\title{
Lakatos' Quasi-empiricism in the Philosophy of Mathematics
}

\author{
Michael J. Shaffer \\ St. Cloud State University
}

\begin{abstract}
Imre Lakatos' views on the philosophy of mathematics are important and they have often been underappreciated. The most obvious lacuna in this respect is the lack of detailed discussion and analysis of his 1976a paper and its implications for the methodology of mathematics, particularly its implications with respect to argumentation and the matter of how truths are established in mathematics. The most important themes that run through his work on the philosophy of mathematics and which culminate in the 1976a paper are (1) the (quasi-)empirical character of mathematics and (2) the rejection of axiomatic deductivism as the basis of mathematical knowledge. In this paper Lakatos' later views on the quasi-empirical nature of mathematical theories and methodology are examined and specific attention is paid to what this view implies about the nature of mathematical argumentation and its relation to the empirical sciences.
\end{abstract}

\section{Introduction}

Imre Lakatos' views on the philosophy of mathematics are important and they have often been underappreciated. The most obvious lacuna in this respect is the lack of detailed discussion and analysis of his 1976a paper and its implications for the methodology of mathematics, particularly its implications with respect to argumentation and the matter of how truths are established in mathematics. ${ }^{1}$ The most important themes that run through his work on the philosophy of mathematics and which culminate in the 1976a paper are (1) the (quasi-)empirical character of mathematics and (2) the rejection of axiomatic deductivism as the basis of mathematical knowledge. When properly understood, the view that Lakatos presented in his 1976a article implies that while the methodology and epistemology of mathematics is a matter of rational thinking (pace the pessimistic irrationalists), it is not rational in the sense that it is constituted by a system of apodictically certain, a priori grounded Euclidean axioms, lemmas, and definitions from which theorems are deductively derived and thereby also secured as certainties.

1 See, for example, Larvor (1998). In Larvor's book Lakatos' 1976a is barely addressed at all. Koetsier (1991) is, however, a notable exception to this point, even though he wrongly argues that Lakatos' 1976a work was incomplete and unsatisfactory due to Lakatos' alleged failure to come to terms with the nature of the falsifiers for mathematical statements. 
Lakatos held that this sort of model of mathematical knowledge is totally artificial and simply misleading. In accordance with his strong Popperian leanings, Lakatos held rather that the advancement of mathematical knowledge is far less rigid and that it is constituted by a sequence of conjectures followed by earnest attempts at refutation via the construction of falsifying counter-examples. So, according to Lakatos, in reality the methodology of pure mathematics is much more like the methodology of the physical sciences than it is like the artifice that is the Euclidean myth. Lakatos is very careful to indicate that this is made manifest simply by looking at the actual history of mathematical achievement. So, he held that our acceptance of the myth of the Euclidean system is simply the result of failing to pay careful enough attention to the history of mathematics as it is actually practiced. ${ }^{2}$ In any case, in this paper Lakatos' later views on the nature of mathematical theories and methodology will be examined and specific attention will be paid to what this view implies about the nature of mathematical argumentation and its relation to the empirical sciences.

\section{Lakatos and the Philosophy of Mathematics}

Imre Lakatos is well-known and well-regarded for his contributions to the philosophy of science. Most notably, he is both well-known and wellregarded for his articulation of the methodology of scientific research programs and his synthesis of methodological and historical approaches to the philosophy of science. His views in these respects were importantly related to the work of Popper, although Lakatos' work on these problems is both novel and important in its own right. Lakatos, of course, also did considerable and deeply interesting work in the philosophy of mathematics that was also based on this essentially Popperian concept of methodology. ${ }^{3}$ Nevertheless, this work has probably received far less attention than it deserves given the long standing problem of understanding the nature of mathematical statements in terms both of their ontological significance and their confirmation. In his sequence of articles in The British Journal for the Philosophy of Science titled "Proofs and Refutations", Lakatos presented a deeply interesting (quasi-)empiricist approach to mathematics and its dynamics based on his 1961 Ph.D. dissertation Essays in the Logic of Mathematical Discovery. ${ }^{4}$ There is little doubt that "Proofs and Refutations" is Lakatos' work on the methodology of mathematics that most philosophers of mathematics are familiar with. However, his posthumously published

\footnotetext{
${ }^{2}$ It is important to note that this theme - one that was clearly initiated by Lakatos has been emphasized more recently in the work of Kitcher (1981 and 1983) and Antonutti 2010, for example.

${ }^{3}$ See Popper (1934/1959).

${ }^{4}$ See Lakatos (1963-4.)
} 
1976 article "A Renaissance of Empiricism in the Recent Philosophy of Mathematics", initially submitted to The British Journal for the Philosophy of Science in 1967, also contains crucial work that importantly extends the views presented in his earlier work. Specifically, in his 1976 article (1976a) Lakatos introduces the crucially important concept of a quasi-empirical theory, and thereby attempts to further establish that mathematical theories, in point of fact, are identifiably empirical in some important sense, while also being importantly different than empirical theories in the natural sciences. In this latter work Lakatos buttressed his earlier 1963-4 view that we cannot adequately explain mathematics as the accumulation of timeless truths via some a priori method. But this critical aspect of Lakatos' views on the methodology of mathematics does not itself provide us with an understanding of the precise sense in which mathematics is supposed to be empirical and how such theories differ from more familiar empirical theories in the natural sciences. However, some of the documents in the Lakatos archive at the London School of Economics and some of his less familiar published writings on these matters allow for the fleshing out of just what he took to be the actual, historically accurate, methodology of mathematics and how it is both similar and different from the properly empirical and experimental sciences.

The typical understanding of Lakatos' view of the methodology of mathematics is clearly derived from his extensive commentary on the matter in Proofs and Refutations. In the first appendix of that book Lakatos explains that the pattern of mathematical discovery takes the following basic form:

(1) Primitive conjecture.

(2) Proof (a rough thought-experiment or argument, decomposing the primitive conjecture into subconjectures or lemmas).

(3) 'Global' counterexamples (counterexamples to the primitive conjecture) emerge.

(4) Proof re-examined: the 'guilty lemma' to which the global counterexample is a 'local' counterexample is spotted. This guilty lemma may have previously remained 'hidden' or may have been misidentified. Now it is made explicit, and built into the primitive conjecture as a condition. The theorem - the improved conjecture supersedes the primitive conjecture with the new proof-generated concept as its paramount new feature (1976b, p. 127).

He adds that this basic methodological pattern is then sometimes followed by the following additional sequence of steps:

(5) Proofs of other theorems are examined to see if the newly found lemma or the new proof-generated concept occurs in them: this concept may be found lying at cross-roads of different proofs, and thus emerge as of basic importance.

(6) The hitherto accepted consequences of the original and now refuted conjecture are checked. 
(7) Counterexamples are turned into new examples - new fields of inquiry open up (1976b, p. 128).

The Lakatosian methodological account of the development of mathematics so understood is interesting in its own right, but it has been discussed much more thoroughly than has his later work, which essentially involves a more detailed epistemological characterization of steps (1)-(3). What his less familiar works allow us to see are the epistemological and methodological features involved in the establishing of mathematical claims and what this shows about their nature.

\section{The Myth of Euclideanism}

First and foremost Lakatos rejected the logical empiricist's view that mathematical knowledge is "a priori, tautologous and infallible (1976a, p. 201)." In various notes and papers Lakatos emphasizes that the traditional Euclidean view of mathematics cannot be correct, especially because many fields of mathematics flourished long before they were formally axiomatized. In a document in archive 4.9 titled "Empiricism in Contemporary Mathematical Philosophy", for example, he explains both that, "...geometry was well developed long before Euclid" and that "...number theory flourished for centuries before it was axiomatized by Dedekind (5)." In response to this recognition Lakatos sought to deploy his own, historically based, concept of the methodology of research programs in pursuit of an acceptable explanation of the methodology and growth of the mathematical sciences. In this same manuscript he explicitly tells us that, “...a new philosophy of mathematics should be oriented on the growth rather than on the foundations of [mathematics] [and] has to take its inspiration in its turn from the history of mathematics $(4.9,4)$." So, an adequate philosophy of mathematics should reflect this fallibility and the sloppier reality of mathematical practice. To this end, in appendix 2 of Proofs and Refutations he explains that,

In deductivist style, all propositions are true and all inferences are valid. Mathematics is presented as an ever-increasing set of eternal, immutable truths. Counterexamples, refutations, criticism cannot possibly enter. An authoritarian air is secured for the subject by beginning with disguised monster-barring and proof-generated definitions and with the fully-fledged theorem, and by suppressing the primitive conjecture, the refutations, and the criticism of the proof. Deductivist style hides the struggle, hides the adventure. The whole story vanishes, the successive tentative formulations of the theorem in the course of the proof-procedure are doomed to oblivion while the end result is exalted into sacred infallibility (1976b, p. 142).

So, Lakatos clearly indicates that the presentation of mathematical knowledge as a deductive Euclidean system is totally artificial, and as such it obscures the actual methodology of mathematics and distorts our understanding of the nature of mathematical claims. The myth of the 
Euclidean methodology of mathematics omits all of the really important details of the actual proof-procedures as they are employed in actual mathematical research that nets real results, and it falsely gives the impression that mathematical methodology involves the a priori deduction of a priori truths from other a priori truths. The Euclidean system is then nothing more than a sanitized fabrication that leaves out the real epistemic procedures of the much more "sloppy" world of the practicing mathematician, and that also misconstrues the nature of mathematical expressions.

\section{The Real Methodology of Mathematics}

So, Lakatos systematically argued that mathematics is itself established by the Popperian method of conjecture and refutation, but not in precisely the same way that physical theories are developed. Rather, mathematics appears to actually develop by the fallible conjecture of "pre-axiomatic" lower-level theorems that are then - and only sometimes - rather artificially knitted into Euclidean-like deductive systems. Moreover, these systems are ultimately empirical in the sense that "the logical flow in such quasi-empirical theories is not the transmission of truth but rather the retransmission of falsity - from special theorems at the bottom ('basic statements') up towards the set of axioms (1976a, 205-206)". In a revealing note in the archive manuscript 4.9 he explains that in 1962 he had used the term 'empiricist' for the general notion of empirical methodology, but that later in 1965 he had replaced this term in his work with the expression 'quasi-empirical' in order to make an important distinction among types of scientific theories. However, of this usage he importantly goes on to add that, "...perhaps the best will be to say 'Popperian' $(4.9,5)$." So, Lakatos' view of the methodology of mathematics is really just an important extension of his modified Popperian methodological account of science from his 1970 to the formal sciences.

In his published 1976a article he importantly explains about this concept of the methodology of meta-mathematics that

...whether a theory is Euclidean or quasi-empirical is decided by the pattern of truth value flow in the system. The system is Euclidean if the characteristic flow is the transmission of truth from the set of axioms 'downwards' to the rest of the system... is quasi-empirical if the characteristic flow is retransmission of falsity from the false basic statements 'upwards' towards the 'hypothesis' (Lakatos, 1976a, p. 206).

So, the real issue is first and foremost one of the structure of the justification that characterizes a particular system. As he explains in his 1976a, it is not what flows qua justification that is important for this distinction (i.e. truth, probability, certain truth, etc.), but rather "... it is the how of the flow that is decisive (206)." He revealingly also tells us that, “...in a Euclidean theory the axioms prove, as it were, the theorems; in a quasi-empirical theory the axioms explain the theorems (m.s., 4.9, 4, see also 1976a, p. 206)." What is 
then curious is that Lakatos tells us that "... a theory which is quasi-empirical in my sense may be either empirical or non-empirical in the usual sense: it is empirical only if its basic theorems are spatio-temporal singular basic statements whose truth values are decided by the time-honored but unwritten code of the experimental scientist (1976a, 206)." So, the crucial issue is then whether or not particular elements of pure mathematics - which are all, according to Lakatos, quasi-empirical - qualify as properly empirical. If they are properly empirical, then they are based on theorems the truth of which can be determined experimentally. In his 1976a and in a document from archive 4.9 titled "Critical Fallibilism Versus Inductive Empiricism in the Philosophy of Mathematics" Lakatos proceeds to explain that "... a Euclidean theory may be claimed to be true; a quasi-empirical theory - at best - to be well-corroborated, but always conjectural (1976a, 206, m.s. 3-4)." As a result, given that we are dealing with a quasi-empirical system, one key issue for Lakatos is then one of the nature of the conjectures constituting the scientific system in question. But, as we shall ultimately see, for Lakatos this is a question about how such conjectures relate to certain other propositions that serve as basic statements in those systems.

The idea is then that, according to Lakatos, mathematics is crucially like empirical science in the sense that its methodology is speculative, imaginative and fallible because of the actual justificational structure of mathematical theories. Both are essentially Popperian and explanatory in character rather than Euclidean in nature. The history of mathematics is then just the history of conjecture and refutation of mathematical theorems and not the derivation of such theorems from indubitable mathematical axioms that are known a priori. As a result, Lakatos' criticism in this respect is most directly a criticism of the classical rationalistic axiomatic approach to the sciences, especially in the case of the formal science of mathematics. However, Lakatos' understanding of mathematics as quasi-empirical does not commit him to the view, as was held by Mill and other more naturalistically minded philosophers, that mathematics is properly empirical. This issue then ultimately turns on the nature of the basic statements that ground a given system of conjectures. Specifically, it concerns the nature of the potential falsifiers of those basic statements (1976a, p. 213). Lakatos tells us that "The 'nature' of a quasi-empirical theory [i.e. whether it is properly empirical or merely quasi-empirical] is decided by the nature of the truthvalue injections into its potential falsifiers (1976a, p. 213)." However he goes on to add that, "Now nobody will claim that mathematics is empirical in the sense that its potential falsifiers are singular spatio-temporal statements (1976a, 213)." Whether or not this claim is in fact correct, there are two important distinctions that are necessary to properly characterize the methodology of mathematics according to Lakatos. First, according to Lakatos, given Euclidean systems at one extreme and what we might call 
"broadly Popperian" or quasi-empirical systems on the other (i.e. those developed at the level of theorems and which proceed by conjecture and refutation) mathematical methodology is of the latter sort. However, the mere rejection of the Euclidean axiomatic method of science is not itself sufficient to properly characterize the methodology of mathematics. In order to properly achieve such a characterization, we also need to recognize the distinction between systems based on basic statements with empirical falsifiers, and those that do not have basic statements with empirical falsifiers. According to Lakatos, mathematics is a system of the latter sort. So, the taxonomy of scientific systems breaks down simply as follows:

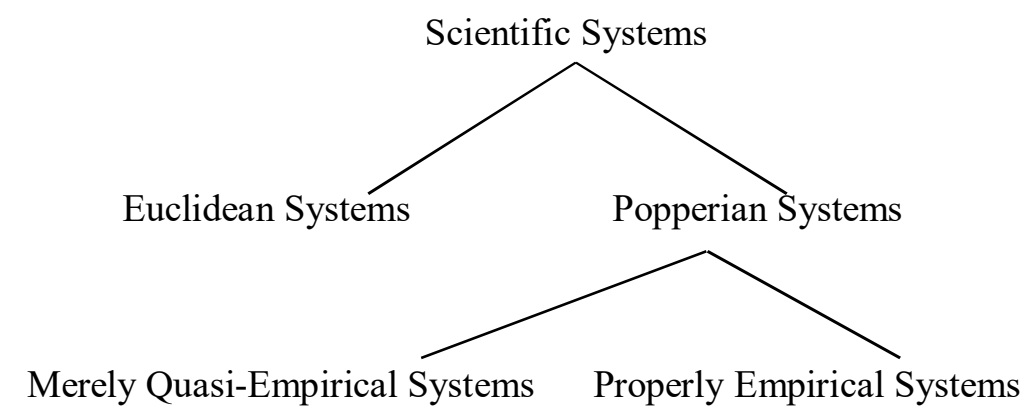

Given this more explicit understanding of the taxonomy of scientific systems, it is then important to note that Lakatos is rather skeptical about the possibility of Euclidean-like axiomatization of many theories (see section 3 of Lakatos, 1976a). More importantly, he staunchly defended the view that the historical emphasis on axiomatization in meta-mathematics is both historically and conceptually anachronistic (m.s. 4.9). What we should grasp from this insightful observation is that the methodology of mathematics is much more like that of the properly empirical sciences simply because the justificational structure of both properly empirical and merely quasiempirical theories is the same and both are not Euclidean in this respect. Of course, those of us who are yet bolder empiricists (especially those who are of a naturalistic bent) would perhaps like to argue that mathematics is itself a properly empirical science.

In any case, it is with respect to this latter issue (i.e. that of the potential falsifiers for mathematical conjectures) that there has been some serious misunderstanding of Lakatos' views on the similarity of mathematics and the properly empirical science. Specifically, Koetsier (1991) argues at length that Lakatos struggled with his understanding of the nature of the potential falsifiers for mathematical statements throughout his work on the methodology of mathematics, and so was not happy with his 1976a. Koetsier says that, "Lakatos has difficulties to point out precisely what are the potential falsifiers in mathematics (1991, p. 62)." Moreover, he also says that, 
"...being unable to locate the mathematical analogon of the singular spatiotemporal statements, Lakatos switches to a different perspective in which theories are compared on the basis of their explanatory power (1991, p. 62)." On this basis Koetsier ultimately claims that, "In 'A Renaissance' [i.e. Lakatos 1976a] Lakatos wanted to show that mathematics is fallible in a way analogous to the fallibility of natural science in Popper's philosophy, but he did not succeed (1991, p. 63)." This critique misses the point that Lakatos was primarily concerned with steps (1)-(3) in his 1976a and that he was more concerned in that work with the structural features of mathematical methodology. But, in his earlier work in $1976 \mathrm{~b}$ he had already settled this issue. The analogs of the singular spatio-temporal statements that serve as falsifiers in properly empirical theories and which serve as falsifiers in mathematics are imaginary mathematical objects. This is clearly the case and the interplay between the counterexamples to the mathematical conjectures that Lakatos considers throughout his $1976 \mathrm{~b}$ are all explicitly described as thought-experiments. Lakatos' $1976 \mathrm{~b}$ is replete with references to the imaginary nature of the potential falsifiers in mathematics (e.g. see 1976b, p. 9 , pp. 74-76 and 86), and this in turn is used also to explain the fallibility of mathematics. Just as in the case of properly empirical theories, mathematical theories can be protected from refutation by treating counterexamples as special cases and then defusing such refutations by directing them away from the core of the theory. This is the whole point of his extensive discussion of monster-barring, local and global counterexamples and testing in 1976b. So, there really is no shift in Lakatos' theory of mathematical methodology from refutation by potential falsifier to purely explanatory considerations. Lakatos clearly holds that the validation of theories in mathematics depends on potential falsification by imaginary cases and that mathematical theories, when successful, are explanatory with respect to those falsifiers. This is admittedly a complex process of accommodation that gives rise to the sort of fallibility that Lakatos famously identified in the properly empirical sciences in his 1970. Thus, Lakatos' philosophy of mathematics is better understood through the lens of his 1976a and 1976b. In 1976b he focuses more attention on the nature of the potential falsifiers in mathematics and in 1976a he focuses more on the structural features of mathematical theories.

\section{Conclusion: The Nature of Mathematical Claims}

What is most interesting about Lakatos' later work on the philosophy of mathematics is its rather radical implications for the epistemology and methodology of mathematics. What his quasi-empiricism implies about mathematical argument and justification is that real, historically accurate, mathematical argument and hence mathematical justification proceeds from the conjecture of theorems to their revision by the use of thought-experiments 
intended to refute such conjectures and not by the derivation of a priori theorems from a priori axioms. So, according to Lakatos, characterizing mathematics in terms of Euclidean systems is a deeply misleading myth that diverts us from seeing that mathematics involves looking for counterexamples that serve as falsifiers. The search for falsifiers in mathematics is just the search for exceptions to initially conjectured theorems by the use of imagination. ${ }^{5}$ But this is only because the truth of such falsifiers cannot (typically) be experimentally established and can only be achieved by thought-experiment. This is in turn true because usually the falsifiers for such conjectures are not singular spatio-temporal statements. As such, our beliefs with respect to mathematical theories are radically fallibilistic and they are justified only in the sense of remaining unrefuted (i.e. they are merely corroborated in the Popperian sense) by imaginary counter-examples. This is of course a radical departure from the view that the results of mathematical investigation are necessary truths derived from a priori axioms, but it is not an unexpected consequence when one recognizes the structural similarity between properly empirical theories and quasi-empirical theories as Lakatos did. What it further suggests is that mathematical argumentation is far less rigorous than is typically assumed and it is bounded at the basic level of falsifiers only by the limits of mathematical imagination. In articulating this position Lakatos establishes that the nature of mathematical truth is a function of how mathematical theories relate to certain types of basic statements that serve as falsifiers for those theoretical claims, just as in the case of the properly empirical sciences. So, in adopting this view Lakatos offered a broadly unified and more realistic account of the methodology of the formal and properly empirical sciences, and that is an important achievement. His view respects the messy details of real mathematical practice and it does not introduce a separate and utterly speculative methodology for mathematics.

\section{References}

Antonutti, M. (2010). Informal Proofs and Mathematical Rigor. Studia Logica, 96, 261-272.

Kitcher, P. (1981). Mathematical Rigor-Who Needs It? Nous, 15, 469-493.

Kitcher, P. (1983). The Nature of Mathematical Knowledge. Oxford: Oxford University Press.

Koetsier, T. (1991). Lakatos' Philosophy of Mathematics. Amstrerdam: North Holland.

\footnotetext{
${ }^{5}$ See Williamson 2016 for a discussion of the epistemology of imagination and its important role in generating knowledge. See also Wansing forthcoming and Priest forthcoming on the same.
} 
Lakatos, I. (1963-4). Proof and Refutations. British Journal for the Philosophy of Science, 14, 1-25, 120-39, 221-43, 296, 342.

Lakatos, I. (1970). Falsification and the Methodology of Scientific Research Programs. In I. Lakatos and A. Musgrave (eds.) Criticism and the Growth of Knowledge. (pp.91-196) Cambridge: Cambridge University Press.

Lakatos, I. (1976a). A Renaissance of Empiricism in the Recent Philosophy of Mathematics. British Journal for the Philosophy of Science 27, 201223.

Lakatos, I. (1976b). Proofs and Refutations. Cambridge: Cambridge University Press.

Larvor, B. (1998). Lakatos. London: Routledge.

Popper, K. (1934/1959). The Logic of Scientific Discovery (Logik der Forschung). London: Hutchinson.

Priest, G. (forthcoming). Thinking the Impossible. Philosophical Studies.

Wansing, H. (forthcoming). Remarks on the Logic of Imagination. Synthese.

Williamson, T. (2016). Knowing by Imagining. In A. Kind and P. Kung (eds.), Knowledge Through Imagination. (pp. 113-123) Oxford: Oxford University Press. 